\title{
Dropping the Pilot
}

\section{Dear Reader,}

Hyundai recently introduced a new transmission that accommodates optimized gear selection based on real-time topological and traffic data. Mimicking human behavior, the vehicle's electronic system uses information going well beyond propulsion and speed to proactively respond to the immediate environment, thereby achieving a higher level of automation. The technology involved is not entirely new, as is evidenced by similar developments from BMW presented in an article in ATZ issue 01/2014 and ZF's use of a similar system to improve fuel-efficiency performance in commercial vehicles.

But Hyundai's approach points the way into the future. To achieve greater efficiency, we need to shift the focus away from individual components and toward holistic systems and process chains. After all, of what use are the arduous incremental gains we make in engine efficiency, of 42,45 , or even $50 \%$, when the source of driving error is sitting behind the steering wheel, slowing to a crawl up a $7.5 \%$ incline, to complete the final $100 \mathrm{~m}$ to the traffic light going 5 to $10 \mathrm{~km} / \mathrm{h}$ in first gear? Sure, they "learned it that way in driving school," but the driving instructor probably had a flat track and a higher gear in mind when talking about anticipatory driving. Even without the additional information about when a traffic light is going to turn green, a vehicle ECU could probably perform the task in a more fuel-efficient manner using optimized speed and gear selection plus a start-stop system, all the more so as all vehicles in the chain would then operate optimally.

Instead of optimizing engines, transmissions and other subsystems, we would be better off optimizing driving perfor- mance. An application-driven focus on holistic systems is already common in many areas of industrial automation, whereas cars, in contrast, are still not designed and engineered using a process-based approach. This focus on the vehicle will need to change if we are to exploit the full potential that electronics and modern control architectures (and AI) offer when it comes to energy efficiency and sustainable solutions. Otherwise we will continue to give away theoretically possible gains through incorrect application in daily use.

All in all, vehicles must become more intelligent to compensate for errors caused by humans who do not always act as appropriately rational regulating factors. Indeed, it is telling that today's automatic transmissions are generally more efficient than their manual counterparts. It's time to delegate authority to someone who can do it better.

We hope you enjoy reading this latest issue!

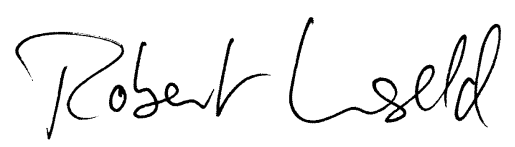

Robert Unseld

Responsible Editor

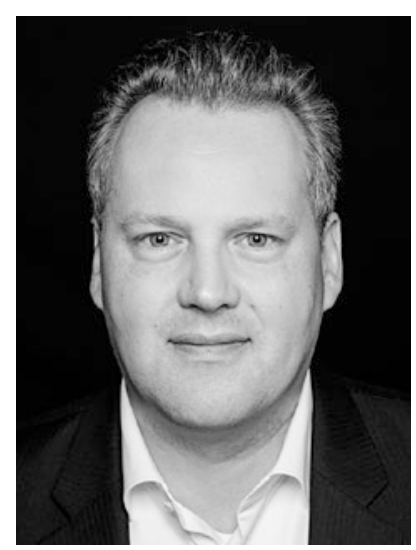



RENT A LOGGER

IPETRONIK's new rental system

- No down payment

- Fixed monthly rent

- Flexible lease term

- Purchase options

- IPEmotion RT included

messtec + sensor masters,

Stuttgart

22.09. - 23.09.2020

Booth 8 A/B 